\title{
Theoretische Homogenitätssehnsucht als Dominanzanspruch
}

Ein Kommentar zu Norman Braun: „Theorie in der Soziologie“1

\section{Von Karl-Siegbert Rehberg}

\author{
„,Von allen Gegensätzen, die die Sozialwissenschaften \\ künstlich spalten, ist der grundlegendste und verderb- \\ lichste der zwischen Subjektivismus und Objektivismus “ \\ (Pierre Bourdieu)
}

\section{I}

Sich der weit verbreiteten Sorge um eine mangelnde Resonanz und Praxisrelevanz der Soziologie anschließend fordert Norman Braun mit Entschiedenheit jene paradigmatische Einheit des Faches, die schon Auguste Comte vorschwebte. Dieser unterschied das „historische“ (also eine Vielfalt unterschiedlichster Ansätze voraussetzen müssende) vom „dogmatischen“ (mit Thomas S. Kuhn zu sprechen: paradigmatischen) Stadium. Wie in diesem enthusiastischen Entwurf der Soziologie als der komplexesten aller Disziplinen, war das 19. Jahrhundert bestimmt durch große umfassende Wissenschaftskonzepte, wie sie in der Nachfolge Charles Darwins von Herbert Spencer oder Ernst Haeckel, in anderen Wissenschaftstraditionen aber auch von Friedrich Engels, Wilhelm Ostwald oder Wilhelm Wundt entwickelt wurden. Nachdem die realen Wissenschaften durch ihre Ausdifferenzierung und den mit ihr verbundenen Modellpluralismus eine derart imaginierteEinheit unwiderruflich zerbrochen haben, verstummt die Klage darüber nicht. Inzwischen ist man selbst in den Naturwissenschaften mit einer Multiperspektivität und Theoriekoexistenz konfrontiert. Gescheitert scheint die Hoffnung, die unbelebte und die belebte Natur und darin wiederum die physischen, psychischen und gesellschaftlichen Zusammenhänge ,aus einem Punkte zu kurieren“ (Goethe, Faust I, Schülerszene), ein nicht willkürlich herbeigezogenes Bild, weil Braun (doch wohl nicht juristisch, sondern therapeutisch gemeint) ein Programm der „Rehabilitation“ soziologischer Theorie entwirft.

Sein Aufsatz, der abermals eine derartige Forderung nach Einheitswissenschaftlichkeit erhebt, hinterlässt zwiespältige Gefühle. Immer neu wiederholte Feindbilder rahmen eine Skizzierung des Kritischen Rationalismus, die im Ganzen als gute und selbstständig weiterentwickelte Studien-Einführung in die wissenschaftstheoretische Tradition seit Carl Gustav Hempel und Paul Oppenheimer, Karl R. Popper, Hans Albert, Imre Lakatos und der Weiterentwicklung in der elementaren Verhaltenstheorie und im Rational Choice gelten kann.

II

Dominant aber ist eine Situationsbeschreibung der Soziologie als heillos heterogener Diskursgemeinschaft, der man nach der Lektüre selbst den Namen „Disziplin“ absprechen müsste, da es ihr intellektuell gerade an dieser zu mangeln scheint. Nicht nur von Braun werden Bilder eines Tribalismus in unvereinbaren Eigenwelten gerne verbreitet, während sich in der Unterschiedlichkeit der Sichtweisen, Begriffe und Methoden doch keineswegs notwendig Frag-

1 Sehr danke ich Matthes Blank für die Unterstützung bei der Verfertigung dieser Kritik-Kritik, sodann den Teilnehmerinnen und Teilnehmern des Mittwochs-Kolloquiums des Dresdner Lehrstuhls für Soziologische Theorie, Theoriegeschichte und Kultursoziologie, in welchem wir den Aufsatz von Norman Braun in eingeübter Offenheit und Bereitschaft auch zu Kontroversen diskutiert haben. Vor allem sind hier wichtige Anregungen von Dominik Schrage, Guido Mehlkop, Tino Heim und Tim Deubel dankbar zu erwähnen. Das Motto ist entnommen: Bourdieu 1993: 49.

Soziale Welt 60 (2009), S. $215-222$ 
mentierung zeigt, vielmehr die unaufhebbare Komplexität des ,Gegenstandes' der Soziologie. Als Vorurteil erweist sich wenigstens die Rede von den unübersetzbaren Stammessprachen, wenn man die Arbeit der Sektionen und Arbeitsgruppen der DGS empirisch analysiert (vgl. Orth / Schwietring / Weiß 2003). Die Rückgriffe auf zentrale Autoren des Faches (und keineswegs nur auf die der je eigenen ,Schule') zeigen einerseits die Weite der Bezugnahmen und verarbeiteten Informationen. Aber ebenso deutlich wird die Präsenz von Hauptautoren in den unterschiedlichsten Kontexten. Man mag das als ,modisch' abkanzeln - aber ,tribalistisch' ist es gewiss nicht.

Derzeitige soziologische Theoriebildung wird durch eine Beschränktheit auf Beschreibungen und Kategorisierungen sowie auf eine ,überwiegend praktizierte Vernachlässigung der Empirie" gekennzeichnet. Dabei seien die Ansprüche vieler Theoretiker nicht gerade bescheiden, fehlten „Theorien mittlerer Reichweite“ im Sinne Robert K. Mertons. Stattdessen dominierten „Großtheorien“ oder als „Theorie“ sich ausgebende Denkansätze, die mit „,eigenwilligen Beschreibungen und Deutungen“ aufträten. Zumeist handele es sich um „,mehr oder weniger erschöpfende Kategorisierungen und Begrifflichkeiten, sowie journalistisch anmutende Zeitdiagnosen“. Man darf raten, welche Lektüren Braun sich zugemutet hat und wen er meinen könnte, wenn er (für ihn) nicht theoriefähige „Theorien“ folgendermaßen vorstellt: Sie seien ,normalerweise beeindruckend weitschweifige, häufig aber wenig präzise theoretische Ausführungen“ mit ,schwammigen Konzepten und Begrifflichkeiten sowie unschlüssigen oder sogar fehlerhaften Argumentationen“.

\section{III}

Obwohl Norman Braun (wie lange vor ihm Vilfredo Pareto) nicht zu Unrecht in vielem, was „Theorie“ heißt, Konstrukte „rein verbaler Natur" sieht, liefert er selbst in der Beschreibung der soziologischen Arbeit doch nur Behauptungen, was dem Autor des „Trattato di Sociologia“ dann doch eher missfallen hätte. Denn alle prinzipiellen Fragen bleiben ungeklärt.

1. Eines der Grundprobleme der Wissenschaften seit der Antike ist ihr Wirklichkeitsbezug. Braun setzt (einer Naturontologie gefährlich nahe kommend) einen „Naiven Realismus“ (Halbfass 1992), welcher von der „Existenz einer Wirklichkeit, die nicht nur eine Vorstellung ist" ausgehe, einem Konglomerat unterschiedlichster Ansätze gegenüber. Deren Zusammenhang ist für ihn in einer ,idealistischen“ [?] Prägung gegeben, wofür er etwa Dekonstruktivismus, Phänomenologie, Semiotik, Strukturalismus und Poststrukturalismus unterschiedslos die Annahme unterschiebt, Wirklichkeit werde „,im Diskurs geschaffen“. Zwar gab es in den ,Wohlstandsgesellschaften' postmoderne Verflüssigungen und spielerische Kontingenzüberziehungen, die es so erscheinen lassen konnten, als bestehe Realität allein aus den menschlichen Konstruktionen. Aber die meisten der genannten Positionen gehen doch eher davon aus, was der ,Erfinder' des „Positivismus“ sowenig wie der „Mythos von Heidelberg“ oder auch Michel Foucault, Jean Baudrillard u.a. bestritten hätten und was die kaum eines „Idealismus“ verdächtigen Soziologen Thomas Luckmann und Peter L. Berger zum Titel eines fachlichen Weltbestsellers gemacht haben: Alle Gegebenheiten, von den Naturzuständen bis zu den faits sociaux, sind immer auch gesellschaftlich konstruiert. Gerade wenn Kultur die der Natur abgerungene, umgearbeitete „Welt“ des Menschen ist, gibt es keine Formen der Vergemeinschaftung und Vergesellschaftung, des Öffentlichen und Intimen, des Ästhetischen und des Technischen ohne Deutung, ist ,das Soziale“ durch und durch kulturell codiert. Weber nannte das, wenn man mir den Bezug auf einen jener toten Autoren, die nach Braun ,heutzutage kaum mehr praktische Bedeutung haben“, nachsehen will: „Kulturbedeutung“. Braun trägt selbst zu einer produktiven Klärung dieser anthropologisch notwendigen Verbindung von Realitätsvoraussetzung und Konstruiertheit nichts bei, sondern belässt es 
bei der behaupteten Unüberwindlichkeit eines Dualismus' von Realismus und Konstruktivismus.

2. Nicht anders steht es mit dem die gesamte Fachgeschichte durchziehenden Verhältnis von Verstehen und Erklären (vgl. Greshoff u.a. 2008). Richtig unterstellt Braun, dass (der nun auch von ihm selbst herangezogene) Weber keineswegs in der neukantianischen Entgegensetzung von naturwissenschaftlichem Erklären und kulturwissenschaftlichem Verstehen befangen blieb, sondern auch als Soziologe an „Warum-Fragen“ interessiert war. Das bedeutet doch wohl, dass er auf deren Beantwortung gespannt gewesen wäre. Und gerade da liegt das Problem. Da auch Braun sich vor elementaren Erläuterungen nicht scheut, darf ich vielleicht auf den selbst im Bachelor-Studium nicht unbekannt bleibenden Sachverhalt verweisen, dass Weber (1976) im berühmten ersten Paragraphen der Soziologischen Grundbegriffe unserem Fach die Aufgabe des Erklärens zwar zuschreibt, gerade dafür ein Verstehen aber zu dessen Voraussetzung machend: „Soziologie [...] soll heißen: eine Wissenschaft, welche soziales Handeln deutend verstehen und dadurch [kursiv von K.-S. R.] in seinem Ablauf und seinen Wirkungen ursächlich erklären will.“ Man sieht also, dass Klassiker-Philologie nicht immer unnütz ist.

3. Es ist ja auch nicht so, dass Soziologen wie die von Braun benannten Ethnomethodologen oder die unter der Rubrik ,verstehende Soziologie“ zusammengefassten Forscherinnen und Forscher die „Notwendigkeit von Erklärungen“ bezweifelten. Gerne würde jeder wohl die kausalen Ursachen und Verkettungen der gegebenen Welt, etwa auch von Prozessdynamiken, aufdecken, und alle Bemühungen in dieser Richtung sind ja durchweg unangefochten. Demgegenüber wird die De-Legitimation anderer Erkenntnismethoden dort, wo der Kausalnexus nicht freizulegen ist, allein von Seiten derer betrieben, die ihrer Methoden so sicher zu sein scheinen. Das legt die Assoziation an den Zusammenhang von „Angst und Methode“ nahe, wie Georges Devereux ihn für die ,Verhaltenswissenschaften“ untersucht hat; im Vorwort dazu beobachtete der amerikanische Anthropologe Weston La Barre, dass die Sozialwissenschaften, die seit dem 17. Jahrhundert „nach dem Prestige der exakten physikalischen Wissenschaften" strebten, sich unverändert nach dem mechanistischen Newtonschen Modell jener Zeit ausrichteten, ,als hätten Einstein und Heisenberg in der Zwischenzeit die Physik nicht revolutioniert" (zit. in: Devereux 1984: 9). Wie anders war das in der Hochzeit einer reflektierten Kultur der Relativität am Anfang des 20. Jahrhunderts, von der auch der wissenssoziologische Relationismus Karl Mannheims inspiriert war. Aber auch damals entfesselte die Zumutung des Einheitsverlustes sofort scharfe Angriffe aus kulturkritischer, geschichtsphilosophischer und szientistischer Sicht.

Zumeist werden methodologische Vereinheitlichungsforderungen mit dem selbstbewussten Anspruch begründet, selbst einer ,erklärenden“ Theorie zu folgen, obwohl diese Prätention oft gar nicht einlösbar ist. Ein Beispiel dafür mögen Analysen sein, die auf dem Axiom der ,rationalen Wahl' aufbauen. Meine These, dass bei aller erkenntnisfördernden Modellierungsarbeit überhaupt nichts ,erklärt“ wird, das nicht vorher verstanden worden wäre, wird dadurch bestätigt, dass ein Rational Choice-Theoretiker wie Hartmut Esser zunehmend mehr unbestimmte, aber prägende Rahmenbedingungen für die Entscheidungsrationalität Einzelner einbeziehen und insofern zu einer Analyse der „Logik der Situation“ kommen musste. Man braucht kulturell produzierte, objektive Sinnsysteme und deren Verstehen, um Handlungsoptionen rekonstruieren und bewerten zu können.

4. Ähnliches gilt für Prognosen, deren Voraussagesicherheit man sich auch wünschen würde. Allerdings ist Skepsis ihrer Reichweite gegenüber angebracht. Der Soziologie wurde nach dem Zusammenbruch des Sowjet-Imperiums oft vorgeworfen, dass sie diesen, wie auch die Implosion der DDR, in keiner Weise vorausgesehen habe (was mitzuteilen übrigens auch die von Braun hoch gelobte ,amerikanische Politologie“ versäumte). Sicher hat 
sich auch hier bewahrheitet, dass die suggestive Kraft des Faktischen und scheinbar Notwendigen den Blick für Alternativen und Umbrüche schwächt. Aber die Frage nach der Prognosefähigkeit der Soziologie erfordert eine wissenschaftstheoretisch reflektiertere Auseinandersetzung, wie beispielsweise Renate Mayntz (1996: 141, 144, 148) sie in großer Klarheit geführt hat: Eine Prognose für Makrophänomene könne, grundsätzlich nicht aufgrund theoretischer Ableitungen“ gegeben werden, denn gesellschaftliche Umbrüche seien ein besonderer Typ sozialer Diskontinuität auf allen Systemebenen bis in den Mikrobereich hinein. Pfadabhängigkeiten schließt das nicht aus, aber es gebe nichtlineare Einflüsse und „Phasensprünge“, sodass gilt: „Je dynamischer, turbulenter und verflochtener das Ganze wird, umso weniger wird, was wir vorhersehen und als nomologische Verallgemeinerung formulieren können“. Das klingt doch vielschichtiger als die Formulierung Brauns, wonach das ,beste Szenario“ für eine prognosefähige Theorie darin bestehe, dass diese ,unabhängig vom jeweiligen Fach [...] sämtliche relevanten Voraussetzungen [...] identifiziert und in kausalen Beziehungen miteinander verknüpft“ und dadurch (wie es, selbst auf eine Zukunftserwartung bezogen, etwas zu normativ heißt) festlege [!], ,wann etwas wo stattfindet oder abläuft“.

\section{IV}

Sehr mag man Norman Braun darin zustimmen, dass die Verbindung der Soziologie mit vielen für sie wichtigen Wissenschaften zerbrochen sei und mehr Interdisziplinarität notwendig wäre. Fraglich allerdings bleibt, ob das an der Selbstbornierung sich abschottender soziologischer Sprachspiel-Gruppen liegt oder an einem Ausdifferenzierungsprozess, der etwa die führende Modellökonomie dazu brachte, ,die Gesellschaft“ weitgehend auszugrenzen - ausgenommen solche Ansätze, die sich einer neoklassischen Zurichtung der Welt einfügen. Schon Alvin W. Gouldner (1974: 118) hatte (selbst-)kritisch von der Soziologie als einer „N+1-Wissenschaft“ gesprochen, welche nur noch das in der akademischen Arbeitsteilung „übriggebliebene ,soziale' Element zu ihrem Bereich“ gemacht habe.

$\mathrm{Zu}$ unterstützen ist ein Programm, das auch die Naturwissenschaften, erst recht die von Braun treffend benannten „Hybriddisziplinen“ (z.B. Demographie und Psychophysik) mit den Kultur- und Sozialwissenschaften wieder in Kontakt brächte, wie das in der ersten Hälfte des 20. Jahrhunderts beispielsweise für die kenntnisreiche Aufnahme der neuesten biologischen Fortschritte durch die Philosophische Anthropologie geschehen war. Das heißt aber nicht, dass man eine naturwissenschaftliche Methodik einfach zu übernehmen hätte, wie dies wohl auch umgekehrt kaum aussichtsreich sein dürfte. Und nicht sollte vergessen werden, dass es eine spezialistische Abwendung von ,großen Fragen“ gerade auch in den neuen lebenswissenschaftlichen Leitdisziplinen gibt. Zwar fehlt es nicht an oftmals dramatisierenden öffentlichen Debatten, etwa um die „Willensfreiheit“ (vgl. Geyer 2004). Im ,Inneren’ dieser Disziplinen gibt es jedoch eine Arbeitsteilung, die sich transdisziplinären Verknüpfungen tendenziell entzieht, schon weil sie für die Kollegen desselben Fachs an einem Hochschulort kaum mehr Chancen zum Austausch bereit hält - wohl aber für Spezialistengruppen auf der ganzen Welt. Fast sehnsüchtig sagte mir jüngst ein Zellbiologe, dass er einen Lehrer hatte, der Konrad Lorenz kannte und sich noch Fragen gestellt habe, wie ich sie an ihn herantrüge. Zwar sind ungewöhnliche disziplinäre Kooperationen nach wie vor erkenntnisfördernd, jedoch dürfte die Hoffnung auf ein einheitliches Paradigma gerade dadurch nicht erfüllt werden.

Man nehme Niklas Luhmanns Adaption des biologischen „Autopoiesis“-Konzeptes als Beispiel: Keineswegs wird damit, wie Braun suggeriert, nur ein „Begriff“ übernommen, vielmehr ein Prozessmodell von Systemen entwickelt. Diese Umstellung der Luhmannschen Theorie hat, weit über die 1940 bei ihrer Formulierung durch Norbert Wiener noch enorm dynamisierend wirkenden kybernetischen Regelkreise hinaus, ihm und seinen Lesern ganz 
neue Wirklichkeitsausschnitte eröffnet. Damit reagierte er auch auf die Übernahme der Kybernetik in politikwissenschaftliche Modelle, denn dort war sie - sei es bei Karl W. Deutsch oder in der staatssozialistischen Hoffnung auf umfassende Steuerung durch die allwissende Partei - nur noch von bedingter Erklärungs- und Konzeptionskraft gewesen. Und doch kam es zu keinem ,einheitlichen Paradigma', weder interdisziplinär, noch innerhalb der Soziologie. Das Modell relativ autonomer interner Komplexitätserhöhung und Selbstergänzung bliebt heuristisch wertvoll, wenn es auch keinerlei methodische Annäherung zwischen den Neurobiologen Humberto R. Maturana und Francisco J. Valera einerseits und dem Bielefelder, in autopoietischer Eigenproduktivität schreibenden Soziologen auf der anderen Seite erzeugte. Maturana (1991: 39 f) hat die Differenz zwischen seinem und dem Ansatz von Luhmann dadurch deutlich zu machen gesucht, dass soziale Systeme, wie beispielsweise Familien, in keiner Weise als Netzwerke der Produktion ihrer eigenen Komponenten aufzufassen seien, vielmehr als eine Form des Zusammenlebens ganz unterschiedlicher Individuen. Zwar mag die Kritik darauf beruhen, dass Maturana nicht anerkennen will, dass die Elemente sozialer Systeme „Kommunikation“ sein sollen. Aber es zeigt dieses Beispiel doch, dass ganz unterschiedliche Konzepte und, wie in der gesamten Wissenschaftsgeschichte nicht selten: Missverständnisse sehr wohl neue Perspektiven eröffnen können, ohne dass irgendeine jener Vereinheitlichungen, die Braun für einen Königsweg hält, erfüllt wäre. Gleichwohl kann jede Bemühung, Kriterien und Regeln für Aussagesysteme, etwa „Theorien mittlerer Reichweite“, und darauf beruhende Prüfverfahren zu formulieren und in der scientific community verbindlich zu machen, die Vergleichbarkeit und Überprüfbarkeit von Forschungsergebnissen und die Anschlussfähigkeit neuer Untersuchungen fördern. Aber daraus ergibt sich die auch von Braun unterstellte Nutzlosigkeit, ja Schädlichkeit unterschiedlicher Ansätze innerhalb der soziologischen Theoriearbeit eben nicht.

\section{V}

Durchgängig wird in Brauns provozieren sollender Streitschrift die Ebene der öffentlichen Geltung der Soziologie als Rezeptionsphänomen vermischt mit den Produktionsbedingungen wissenschaftlicher Theoriebildung, worin man einen schwerwiegenden Denk- und Kategorienfehler sehen darf. Durchaus handelt es sich in beiden Fällen um Geltungszusammenhänge, aber doch um sehr verschiedene. Die öffentliche Rezeption folgt eben nicht wissenschaftstheoretischen Erwägungen. Das Argument einer ,Wirkungslosigkeit' der deutschen Soziologie ist überdies längst zum Stereotyp geworden. Kein Soziologiekongress, der nicht in den führenden Zeitungen die Klage darüber auslöste, dass das einstmals so tief in die Gesellschaft hineinwirkende Fach heute eher zur langweiligen normal science geworden sei. Der 34. DGS-Kongress 2008 in Jena blieb davon verschont, weil sein Titel „Unsichere Zeiten“" angesichts des nur drei Wochen zurückliegenden, folgenreichen Zusammenbruchs der Investmentbank Lehman Brothers Inc. wie gerufen kam. Üblicherweise jedoch wird diese missmutige Beobachtung vor allem in Blättern geäußert, welche die neomarxistische und durch die Kritische Theorie inspirierte Gesellschaftstheorie während der Studentenrevolte keineswegs mit Zuspruch begleitet hatten. Und wenn Norman Braun moniert, die Soziologie habe „kaum noch gesellschaftliche Auswirkungen“, dürfte er unser Fach wohl kaum in jene Zeiten zurückversetzen wollen.

Übrigens scheint ein melancholisch-gekränkter Blick auf die eigene Disziplin kein deutsches Spezifikum zu sein; in allen Beschwörungen der chronischen Krisenhaftigkeit der Soziologie spiegelt sich nicht nur das Land des „tragischen Bewusstseins“. Liest man die wissenschaftsprogrammatischen Presidential Addresses der American Sociological Association (vgl. Eichler 1998: 5ff), hat sich dort eine große Anzahl der Vorsitzenden skeptisch gegenüber dem Stand der von ihnen repräsentierten Wissenschaft und ihren Möglichkeiten geäußert. Die emphatische Hoffnung Lester Wards im Jahr 1907 - ,we have come to know what 
society really is" - wird überdeckt von Selbstzweifeln. Es wäre wohl kein Vorsitzender der Deutschen Gesellschaft für Soziologie auf den Gedanken gekommen, zu der (gerade in diesem Jahr stattfindenden) hundertsten Wiederkehr ihres Bestehens das Motto „Accounting for the Rising and Declining Significance of Sociology" auszugeben, wie die ASA das zu ihrem 100th Annual Meeting 2005 in Philadelphia tat. Und die Resonanzschwäche der amerikanischen Soziologie wurde zumindest zweimal offiziell thematisiert, zuletzt 2004, als Michael Burawoy eine „Public Sociology“ forderte.

Ohne Selbstberuhigung sollte man mit Blick auf Experteneinfluss oder Pressepräsenz die Verwendung soziologischen Wissens differenzierter darstellen als dies oft geschieht. Etwa gibt es einen journalistischen Alltagsbedarf nach soziologischen Deutungen jedweden Phänomens. Und wenn auch unbestritten sein mag, dass es unterschiedliche Präsentations-Begabungen und eine ungleich verteilte Publizität, im besten Fall sogar die Rolle „öffentlicher Intellektueller" gibt, werden sich das Fach und die DGS immer neu darum bemühen müssen, soziologische Forschungsresultate in die Öffentlichkeit zu bringen. Jedoch ist die publizistische Wahrnehmung sozialwissenschaftlicher Forschungsleistungen auch dadurch mitgeprägt, dass es eine verlässliche Berichterstattung über sie kaum gibt. Während man als Laie von Mondgestein, Klimafaktoren, Enzymen, Zellmanipulation, von Vulkanen auf dem Meeresboden oder Gletscherverschiebungen manches zu hören bekommt, hat die gesamte Sachforschung in unserem Feld kaum eine verlässliche Chance medialer Wahrnehmung, auch wenn einzelne Publizisten sich darum durchaus bemühen mögen. Stattdessen dominiert in den meinungsbildenden Feuilletons eine subtile und intellektuell bereichernde Parallel-Rhetorik, die mit konkurrierenden Deutungsansprüchen auftritt. Darüber sollten wir glücklich sein, denn in den meisten Ländern existiert derlei nicht. Aber die Hoffnung, die Umstellung auf eine Einheitsmethodik würde über Prominenz oder Vergessen einer Disziplin entscheiden, dürfte wohl eher naiv sein. Und bei aller Bewunderung für die Modellierungsraffinesse aller auf Gesetzmäßigkeiten zielenden soziologischen Ansätze wird man wohl doch nicht übersehen können, dass es zu gesellschaftlicher Wirksamkeit viel eher Autoren gebracht haben, denen Braun vorwirft, nur ,philosophisch angehauchte“ (man spürt geradezu den ,Odem', wenn nicht Gottes zumindest der Eingebung) Texte und Kategorienschemata entwickelt zu haben. Die Beispielsnamen sind allen vertraut, etwa - um die Gründungsväter dahingestellt sein zu lassen - Theodor W. Adorno, Talcott Parsons, Erving Goffman, C. Wright Mills, Niklas Luhmann, Ralf Dahrendorf, Anthony Giddens, Pierre Bourdieu (Foucault will ich dem Soziologiekritiker ersparen), später Ulrich Beck, Oskar Negt, Hans Joas, Wilhelm Heitmeyer oder Heinz Bude, nicht zu vergessen Jürgen Habermas als weltweit anerkannter Personifikation , großer Theorie'. Aber, wie gesagt, das alles sind keine Geltungskriterien für Wahrheit, den Stand der Forschung oder den Beitrag einer Disziplin zur Wirklichkeitsdurchdringung.

\section{VI}

Gegen Norman Brauns These meine ich, dass Soziologie als „Wirklichkeitswissenschaft“ einer Vielzahl theoretischer Ansätze bedarf, d.h. als monoparadigmatische Spezialdisziplin, deren höchste Belohnung in der Präsenz in reviewed journals besteht, aussichtslos wäre. Letztere mag zwar geeignet sein, Berufungsverfahren zu beschleunigen, da Quantität und Publikationsorte das Lesen ersetzen können. Aber ein pragmatischer Reduktionismus ist noch keine ,Einheit'. Wer diese will, sollte übervereinfachte Oppositionen ebenso vermeiden, wie aggressive Lagerbildungen. Braun hat recht darin, dass er einen nomologischen Theorietypus deutlich absetzt von anderen Denkbemühungen des Faches. Diese bestehen jedoch keineswegs nur aus assoziativen Erzählungen und unüberprüfbaren Behauptungen. Wenn die Moderne als Epoche eines legitimen Wertpluralismus angesehen wird, so gilt das auch für die in ihr entwickelten Formen wissenschaftlicher Weltwahrnehmung. Da Braun trotz seiner Ab- 
lehnung jeden philosophischen ,Ahnenkultes' selbst auf große Zeugen der Vergangenheit nicht ganz verzichtet, wenn es darum geht, die ,allgemeine Akzeptanz" des individualistischen Ansatzes durch schmückende Namen wie David Hume oder Adam Smith zu plausibilisieren, sei nochmals erlaubt, auf Max Weber zurückzugreifen. Dieser Kantianer, den die Unerkennbarkeit des „Wesens“ der Geschichte, der Gesellschaft oder anderer Ausformungen des „Dings an sich“ zur asketischen Selbstbeschränkung, also zu Perspektivierungen und einer mühevollen Objektivierung von Wirklichkeitsausschnitten, zwang, hat durchgängig gegen die simple Gegenüberstellung von Realitätsleugnung und Einheitsmethodik gekämpft und ,strenge Wissenschaftlichkeit“" gefordert - selbst wenn man nicht glaubt, Gesetzmäßigkeiten finden zu können.

Von diesem Autor wäre zudem zu lernen, dass nicht nur die empirische Gegenwartsanalyse im Dienste des Verstehens der eigenen Zeit steht, sondern ganz ebenso eine historische Empirie, welche allerdings nur in engster Zusammenarbeit mit den Geschichtswissenschaften (einschließlich aller Spezialhistoriographien wie der Kunst- oder Wissenschaftsgeschichte) methodische Sicherheit gewinnen kann. Ebenso ist eine andere Steigerung soziologischer Beobachtungskomplexität unverzichtbar: Auch innerhalb der im engeren Sinne empirischen Methoden sollten ,quantitative' und ,qualitative' Vorgehensweisen einander ergänzen, während durch ein einseitiges Untersuchungs- und Datenprüfungsideal allzu oft ein unüberbrückbarer Gegensatz zwischen diesen geschaffen wird. So erschien es mir keineswegs als Sieg der Wissenschaftlichkeit oder der Professionalität, als unter der Dominanz quantitativer Sozialforscher 2003 die qualitativ Arbeitenden sich innerhalb der DGS in eine eigene Sektion flüchten mussten - und dies in einem Augenblick, in dem in der Forschungspraxis Methodenmix geradezu gefordert ist.

\section{VII}

Denkt man an die Leitwissenschaften, die Norman Braun zum Vorbild dienen, so mag eine Nebenbeobachtung überraschen: Die sie verbindende Grundstruktur des Denkens mutet den Wissenschaftlerinnen und Wissenschaftlern häufig eine Glaubensintensität zu, derer man in der distanzierten soziologischen Betrachtung eigentlich kaum bedürfte. Es gilt dies vor allem für anthropologische Rationalitätsaxiome, die den ökonomischen und verhaltensorientierten Theorien zugrunde liegen. So darf es vor allem überraschen, dass Norman Braun seine Empfehlung, ,der Ökonomik zu folgen“, gerade in einer Zeit gibt, in der eine globale Finanzkrise die Weltwirtschaft trifft, ohne dass eine Wirtschaftswissenschaft, welche die Monetarisierung von immer mehr Lebensbereichen, die Ausschaltung des Staates als Kontrollinstanz und die umfassende Selbststeuerung von Märkten propagiert hatte, irgendwelche prognostische Leistungen über die Folgen ihrer Empfehlungen beigetragen hätte. Obwohl erste Ansätze eines ökonomischen Neo-Institutionalismus kulturelle Faktoren und soziale Rahmenbedingungen des Wirtschaftens stärker wieder ins Bewusstsein hoben, schienen die Hauptrezepte der von Braun als theoretisch so exakt angesehenen Neoklassik unverändert zu gelten - eine Suggestion, der etwa auch die rot-grüne Bundesregierung weitgehend erlegen war, wenn es auch nicht so schlimm kam, wie in der Verheerung Chiles durch die Chicago Boys.

Abschließend sei auf etwas Stilistisches verwiesen: Das Grauen Norman Brauns vor Weitschweifigkeit hätte ihn dazu bringen sollen, die vielen Redundanzen in einer relativ knappen Argumentation einzudämmen - aber vielleicht ist das ein ästhetisches Argument und insofern jenseits aller Theorie. 


\section{Literatur}

Bourdieu, Pierre (1993): Sozialer Sinn. Kritik der theoretischen Vernunft [frz. zuerst 1980], Frankfurt / Main.

Eichler, Margrit (1998): Making Sociology More Inclusive, in: Current Sociology 46 / 2, S. 5-28.

Devereux, Georges (1984): Angst und Methode in den Verhaltenswissenschaften [frz. zuerst 1967], Frankfurt / Main.

Geyer, Christian (Hrsg.) (2004): Hirnforschung und Willensfreiheit. Zur Deutung der neuesten Experimente, Frankfurt / Main.

Gouldner, Alvin W. (1974): Die westliche Soziologie in der Krise, Reinbek.

Greshoff, Rainer / Georg Kneer / Wolfgang Ludwig Schneider (Hrsg.) (2008): Verstehen und Erklären. Sozial- und kulturwissenschaftliche Perspektiven, München.

Halbfass, Wilhelm (1992): Naiver Realismus, in: Joachim Ritter / Karlfried Gründer (Hrsg.), Historisches Wörterbuch der Philosophie, Band 8, Darmstadt, Sp. 160-161.

Mayntz, Renate (1996): Gesellschaftliche Umbrüche als Testfall soziologischer Theorie, in: Lars Clausen (Hrsg.), Gesellschaften im Umbruch. Verhandlungen des 27. Kongresses der Deutschen Gesellschaft für Soziologie in Halle an der Saale 1995, Frankfurt / Main - New York, S. 140-153.

Orth, Barbara / Thomas Schwietring / Johannes Weiß (Hrsg.) (2003): Soziologische Forschung. Stand und Perspektiven. Ein Handbuch, Opladen.

Riegas, Volker / Christian Vetter (1991): Zur Biologie der Kognition. Ein Gespräch mit Humberto R. Maturana und Beiträge zur Diskussion seines Werkes, Frankfurt / Main.

Weber, Max (1976): Wirtschaft und Gesellschaft. Grundriss der verstehenden Soziologie [zuerst 1920]. 5., rev. Aufl., hrsg. v. Johannes Winckelmann, Tübingen.

Prof. Dr. Karl-Siegbert Rehberg Technische Universität Dresden Institut für Soziologie Chemnitzer Straße 46 a 01062 Dresden Karl-Siegbert.Rehberg@tu-dresden.de 